\title{
SPLIT FACES AND IDEAL STRUCTURE OF OPERATOR ALGEBRAS
}

\author{
HARALD HANCHE-OLSEN
}

\section{Introduction and notation.}

The study of facial vs. ideal structure in operator algebras was initiated in 1963 by the independent works of Effros [10] and Prosser [14]. They found a one-to-one correspondence between norm closed left ideals in a $\mathrm{C}^{*}$-algebra, norm closed faces in its positive cone, and weak*-closed faces of its state space. In this correspondence, two-sided ideals correspond to invariant faces.

However, Effros and Prosser failed to characterize the invariant faces in a purely geometric way. In [16; Thm. 3.2] Størmer proved that these were exactly the Archimedean faces, while Alfsen and Andersen introduced the concept of a split face and noted that invariant faces are split [2; Prop. 7.1].

In section 2 we generalized to JB-algebras the correspondence between twosided ideals and split faces. (For the theory of JB-algebras and their ideals, see [7], [13], [15], [3; § 2], [8], and [9]). At the same time, and equally important, we get new and more direct proofs of known results for $\mathrm{C}^{*}$-algebras. The reader primarily interested in $\mathrm{C}^{*}$-algebras may substitute $\mathrm{C}^{*}$-algebras and two-sided ideals for JB-algebras and Jordan ideals in section 2. By trivial modifications in the proofs, she can then make them valid for the $\mathrm{C}^{*}$-algebra case.

It should be mentioned here that all the results of section 2 are due to E. M. Alfsen and F. W. Shultz (unpublished). We would like to thank Alfsen and Shultz for their kind permission to include this material.

Section 3 contains the main new result of this paper. We define the structure space Prim $(K)$ for an arbitrary compact convex set $K$, and give necessary and sufficient conditions for the canonical surjection $\partial_{e} K \rightarrow$ Prim $(K)$ to be open.

In section 4 we apply Theorem 3.1 together with the results of section 2 to generalize to JB-algebras Glimm's result [12], that the canonical mapping $\partial_{e} K \rightarrow \operatorname{Prim}(\mathscr{A})$ is open when $\mathscr{A}$ is a $C^{*}$-algebra with state space $K$. The proof is rather different from Glimm's original proof, because of the lack of inner automorphisms.

By a Jordan ideal in a JB-algebra $A$ we shall mean a subspace $J$ such that, whenever $a \in A$ and $b \in J$, then $a \circ b \in J$. Jordan ideals correspond to two-

Received February 15, 1980. 
sided ideals in the following strict sense: A norm closed self-adjoint complex subspace $\mathscr{I}$ of a $C^{*}$-algebra $\mathscr{A}$ is a two-sided ideal iff its self-adjoint part $\mathscr{I}_{\text {sa }}$ is a Jordan ideal of $\mathscr{A}_{\mathrm{sa}}$. This can be seen either by considering the weak*-closure in $\mathscr{A}^{* *}$ of $\mathscr{I}$ and using [8; Thm. 2.3], or by appealing to [11; Thm. 2].

If $a$ is an element of a JB-algebra $A$ and $\varrho$ is a linear functional on $A$, we denote by $\langle a, \varrho\rangle$ the value of the functional $\varrho$ at the element $a$. Note that any JBW-algebra is canonically order-and norm-isomorphic to the space $A^{b}(K)$ of bounded affine functions on its normal state space $K$.

We define the annihilators of a subset $J$ of a JB-algebra $A$ and a subset $F$ of its state space $K$ by

$$
\begin{aligned}
& J^{\perp}=\{\varrho \in K:\langle a, \varrho\rangle=0 \text { for all } a \in J\} \\
& F_{\circ}=\{a \in A:\langle a, \varrho\rangle=0 \text { for all } \varrho \in F\} .
\end{aligned}
$$

Similarly, we define the annihilator $J_{\perp}$ of a subset $J$ of the JBW-algebra $M$ and the annihilator $F^{\circ}$ of a subset $F$ of its normal state space $K$.

If $a, b$ are elements of a JB-algebra $A$ we define their Jordan triple product $\{a b a\}$ by

$$
\{a b a\}=2 a \circ(a \circ b)-a^{2} \circ b .
$$

If $\varrho$ is a functional on $A$, we define functionals $a \circ \varrho$ and $\{a \varrho a\}$ by the formulas,

$$
\begin{aligned}
\langle b, a \circ \varrho\rangle & =\langle a \circ b, \varrho\rangle, \\
\langle b,\{a \varrho a\}\rangle & =\langle\{a b a\}, \varrho\rangle .
\end{aligned}
$$

Note that if $\varrho$ is positive, then $\{a \varrho a\}$ is positive.

Acknowledgement. We would like to thank E. M. Alfsen for many fruitful discussions.

\section{Split faces.}

Let $K$ be a convex set. A face $F$ of $K$ is called a split face $[1 ; \S$ II.6] if there exists a face $F^{\prime}$ such that $K$ is a direct convex sum of $F$ and $F^{\prime}$ in the following sense: Any $\varrho \in K$ can be written as

$$
\varrho=\lambda \sigma+(1-\lambda) \sigma^{\prime},
$$

where $\lambda \in[0,1]$ is unique, and $\sigma \in F$ (respectively $\sigma^{\prime} \in F^{\prime}$ ) is unique (except for the case $\lambda=0$ (respectively $\lambda=1$ )).

Note that the face $F^{\prime}$ is uniquely determined by $F$. It is called the complement of $F$. Also, the mapping $\varrho \rightarrow \lambda$, where $\lambda$ is determined by (2.1), is a bounded affine function in $K$ which has $F$ as its peak set. In our applications $K$ will be 
the base in a base-norm space $(E, K)[1 ; \mathrm{p} .77]$. Then the above affine function on $K$ extends to a bounded linear functional on $E$. Hence, split faces are norm exposed and, in particular, norm closed.

The following result is included in [4; Thm. 11.5], but the present proof makes no use of the machinery of [4].

THEOREM 2.1. Let $M$ be a JBW-algebra and $K$ its normal state space. There is a one-to-one correspondence between split faces $F$ of $K$ and central projections $e$ in $M$, given by:

(i) $F=\{\varrho \in \mid\langle e, \varrho\rangle=0\}$

(ii) $e$ is the unique affine function in $K$ which is identically 0 on $F$ and 1 on $F^{\prime}$.

Proof. Let $F$ be a split face of $K$. Define $e$ to be the affine function $\varrho \rightarrow$ $1-\lambda$, where $\lambda$ is the scalar occuring in (2.1). It is easily seen that $e$ is an extreme point in the positive unit ball of $M$, and hence a projection. We have to show that $e$ is central.

To this end consider an arbitrary element $a \in A$. If $\varrho \in F$ we find, using the Cauchy-Schwarz inequality, that

$$
|\langle e \circ a, \varrho\rangle|^{2} \leqq\langle e, \varrho\rangle\left\langle a^{2}, \varrho\right\rangle=0 .
$$

Thus the affine function $e \circ a$ vanishes on $F$. Similarly, $(1-e) \circ a$ vanishes on $F^{\prime}$, so $e \circ a$ coincides with $a$ on $F^{\prime}$. Repeating the argument, we find that the same holds for $\{e a e\}=2 e \circ(e \circ a)-e \circ a$. Since an affine function on $K$ is determined by its restrictions to $F$ and $F^{\prime}$, we conclude that $e \circ a=\{e a e\}$. Thus $e$ is central by [7; Lemma 2.11].

The proof that, conversely, a central projection $e$ determines a split face by (i) is left to the reader.

Combining Theorem 2.1 with [8; Thm. 2.3] we immediately obtain

COROLlary 2.2. There is a one-to-one correspondence between weak*-closed Jordan ideals $J$ of $M$ and split faces $F$ of $K$, given by $F=J_{\perp}$ and $J=F^{\circ}$.

Indeed, when the central projection $e$ corresponds to the split face $F$, we have $J=\{e M e\}$.

Passing to the duality of a JB-algebra and its dual, we have:

Theorem 2.3. Let $A$ be a JB-algebra and $K$ its state space. There is a one-toone correspondence between norm closed Jordan ideals $J$ of $A$ and weak*-closed split faces $F$ of $K$, given by $F=J^{\perp}$ and $J=F_{0}$. 
Proof. If $J$ is a norm closed Jordan ideal of $A$, then $J^{\perp}$ is a split face and $J$ $=\left(J^{\perp}\right)_{0}$. This is a trivial consequence of Cor. 2.2 and the Hahn-Banach separation theorem. (Consider the weak*-closed ideal $\bar{J}$ in $A^{* *}$ ).

Conversely, that $F_{\circ}=F^{\circ} \cap A$ is a Jordan ideal when $F$ is a split face also follows trivially from Cor. 2.2. That $F=\left(F_{\circ}\right)^{\perp}$ follows, for example, from [1; Thm. II.6.15]. A more elementary proof is the following: Note that the unit ball of $\operatorname{lin} F$ is $\operatorname{co}(F \cup-F)$. By the Krein-Smulian theorem it follows that lin $F$ is weak*-closed. If $\varrho \in K-F$, we can then separate $\varrho$ from lin $F$ with some $a \in A$. Then $a \in F_{\mathrm{o}}$, and so $\varrho \notin\left(F_{\mathrm{o}}\right)^{\perp}$. This completes the proof.

Our next is a generalization of [10; Cor. 6.2].

THEOREM 2.4. Let $F$ be a split face of the state space $K$ of a JB-algebra A. Then its weak*-closure $\bar{F}$ is also a split face of $K$.

Proof. By Cor. 2.2, $F_{\circ}=F^{\circ} \cap A$ is a Jordan ideal of $A$, and hence $G=\left(F_{\circ}\right)^{\perp}$ is a weak*-closed split face of $K$. We shall prove that $\bar{F}=G$.

Let $e$ be the central projection in $A^{* *}$ such that $F^{\circ}=(1-e) \circ A^{* *}$. Since $G_{\circ}$ $=F^{\circ} \cap A$, the mapping $a \rightarrow e \circ a$ induces an injective, and hence isometric, homomorphism $A / G_{\circ} \rightarrow e \circ A^{* *}$.

Let $a \in A$. As in the proof of Theorem 2.1, we note that $e \circ a$ is the unique affine function on $K$ coinciding with $a$ on $F$ and vanishing on $F^{\prime}$. Therefore,

$$
\|e \circ a\|=\sup \{|\langle a, \varrho\rangle|: \varrho \in F\} \text {. }
$$

On the other hand, the quotient norm of $a+G_{\circ}$ in $A / G_{\circ}$ satisfies

$$
\left\|a+G_{\circ}\right\| \geqq \sup \{|\langle a, \varrho\rangle|: \varrho \in G\} .
$$

Since $\left\|a+G_{\circ}\right\|=\|e \circ a\|$, an application of the Hahn-Banach separation theorem yields $G \subseteq \bar{F}$.

Finally, we mention a geometric property of Jordan homomorphisms:

Proposition 2.5. Let $M_{1}$ and $M_{2}$ be $\mathrm{JBW}$-algebras with normal state spaces $K_{1}, K_{2}$ respectively. If $\varphi: M_{1} \rightarrow M_{2}$ is a weak*-continuous Jordan homomorphism, then the predual map $\varphi_{*}$ maps split faces of $K_{2}$ onto split faces of $K_{1}$.

Proof. We only scetch the proof, since this result is not needed in the sequel. Let $F$ be a split face of $K_{2}$. We claim

$$
\varphi_{*}(F)=\varphi^{-1}\left(F^{\circ}\right)_{\perp},
$$

which will complete the proof, by Cor. 2.2. 
The special case

$$
\varphi_{*}\left(K_{2}\right)=\operatorname{Ker}(\varphi)_{\perp}
$$

is, in fact, easily proved using the Hahn-Banach extension theorem. This special case then yields the general case when we consider the composition of $\varphi$ with the canonical map $M_{2} \rightarrow M_{2} / F^{\circ}$.

\section{Structure space of an arbitrary compact convex set.}

In this section $K$ will be a compact convex set in a locally convex topological vector space. Given $\varrho \in \partial_{e} K$ there exists a smallest closed split face $\bar{F}_{e}$ containing $\varrho$. (See $[1 ;$ p. 146]. Note that our notation differs from that in [1]. We write $\bar{F}_{e}$ although in this generality we attach no meaning to the symbol $F_{\varrho}$. This is for consistency with the notation of section 4). We call the split face $\bar{F}_{e}$ primitive, and denote by Prim $(K)$ the set of all primitive split faces. We endow Prim $(K)$ with the structure topology, whose closed sets are those of the form

$$
\{G \in \operatorname{Prim}(K): G \subseteq F\},
$$

where $F$ is a closed split face of $K$. This topology exists by virtue of [1; Prop. II. 6.20]; we remark that Størmer's axiom, as imposed in [1; Lemma 6.25] is not necessary for this definition.

We consider the map $\varrho \rightarrow \bar{F}_{e}$ of $\partial_{e} K$ onto Prim $(K)$. This mapping is continuous, with $\partial_{e} K$ given the relative topology. We will characterize those $K$ for which this map is also open. First, however, we need a definition.

Following [1; p. 146] we say that $K$ satisfies Stormer's axiom if, whenever $\left(F_{\alpha}\right)$ is a collection of closed split faces of $K$, the closed convex hull $\overline{c o}\left(\bigcup_{\alpha} F_{\alpha}\right)$ is a split face.

The following Theorem is an improvement of [1; Lemma II.6.29]. Note that we do not use the concept of sufficiently many inner automorphisms, which was used in [1] and is also buried in Glimm's original proof of the corresponding $\mathrm{C}^{*}$-algebra result $[12]$.

THEOREM 3.1. Let $K$ be a compact convex set in a locally convex topological vector space. The mapping $\varrho \rightarrow \bar{F}_{e}$ is open from the relative topology of $\partial_{e} K$ to the structure topology of Prim $(K)$ iff $K$ satisfies Stormer's axiom and the following condition:

$\left(^{*}\right)$ For any $G \in \operatorname{Prim}(K)$, the set $\left\{\varrho \in \partial_{e} G: \bar{F}_{e}=G\right\}$ is dense in $\partial_{e} G$.

Proof. 1. Assume that the map $\partial_{e} K \rightarrow \operatorname{Prim}(K)$ is open. Let $\left(F_{\alpha}\right)$ be a collection of closed split faces of $K$, and consider the following (relatively) open subset of $\partial_{e} K$ : 


$$
V=\partial_{e} K-\overline{\bigcup_{\alpha} \partial_{e} F_{\alpha}}
$$

By assumption the set $\left\{\bar{F}_{\varrho}: \varrho \in V\right\}$ is open in Prim $(K)$. By definition of the structure topology, there exists a closed split face $F$ of $K$ such that, whenever $\varrho \in \partial_{e} K$ :

$$
\varrho \notin F \Leftrightarrow \bar{F}_{\varrho}=\bar{F}_{\sigma} \text { for some } \sigma \in V .
$$

If $\varrho \in \partial_{e} F_{\alpha}$ then $\bar{F}_{\varrho} \subseteq F_{\alpha}$, and so, by (3.1), $\bar{F}_{\varrho} \neq \bar{F}_{\sigma}$ for all $\sigma \in V$. By (3.2), $\varrho \in F$, and therefore $F_{\alpha} \subseteq F$. We claim that $F=\overline{c o}\left(\bigcup_{\alpha} F_{\alpha}\right)$. If not, we find some $\varrho \in \partial_{e} F$ with $\varrho \notin \overline{\operatorname{co}}\left(\bigcup_{\alpha} F_{\alpha}\right)$. By (3.1) $\varrho \in V$, so by (3.2) $\varrho \notin F$. This contradiction proves our claim, and the validity of Størmer's axiom is proved.

Next, assume that $\left({ }^{*}\right)$ does not hold and choose $G \in \operatorname{Prim}(K)$ not satisfying ${ }^{*}$ ). Then there exists an open set $V \cong \partial_{e} K$ such that $V \cap G \neq \varnothing$ and $G \neq \bar{F}_{e}$, whenever $\varrho \in V$. As above, there is a closed split face $F$ of $K$ such that (3.2) holds. If $G=\bar{F}_{Q}$ then, by (3.2), $\varrho \in F$ and so $G \cong F$. By (3.2) this implies that $G \cap V=\varnothing$, which is a contradiction. Thus $\left(^{*}\right)$ is necessary.

2. Assume that $K$ satisfies Størmer's axiom and the property $\left({ }^{*}\right)$. Let $V$ be a (relatively) open subset of $\partial_{e} K$, and let

$$
F=\overline{c o}(\bigcup\{G \in \operatorname{Prim}(K): G \cap V=\varnothing\}) .
$$

By Størmer's axiom, $F$ is a split face. We claim that

$$
\left\{\bar{F}_{\varrho}: \varrho \in V\right\}=\{G \in \operatorname{Prim}(K): G \Phi F\},
$$

which will complete the proof since the righthand side of (3.4) is an open subset of $\operatorname{Prim}(K)$.

Milman's theorem implies that the union of all $\partial_{e} G$, where $G \in \operatorname{Prim}(K)$ and $G \cap V=\varnothing$, is dense in $\partial_{e} F$. In particular, since $V$ is open, $V \cap \partial_{e} F=\varnothing$. Thus, if $\varrho \in V$ then $\bar{F}_{Q} \Phi F$ and one inclusion in (3.4) is proved.

On the other hand, if $G \in \operatorname{Prim} K$ and $G \Phi F$ then (3.3) implies that $G \cap V$ $\neq \varnothing$. By the property $\left({ }^{*}\right), G=\bar{F}_{Q}$ for some $\varrho \in G \cap V$. Now the second inclusion in (3.4) follows, and the proof is complete.

Remark. If $K$ is a Choquet simplex, any extreme point of $K$ is a split face, and so the property $\left({ }^{*}\right)$ is trivial. However, $K$ does not satisfy Størmer's axiom unless $\partial_{e} K$ is closed [1; Thm. II.7.19]. Thus $\left({ }^{*}\right)$ does not imply Størmer's axiom.

To see that, conversely, Størmer's axiom is not sufficient in the above Theorem, we consider a compact convex set $K$ which contains only one nontrivial closed split face $F$. Then Størmer's axiom is trivially satisfied. If $F$ contains an extreme point $\varrho$ which is isolated in $\partial_{e} K$, then $\{\varrho\}$ is an open subset of $\partial_{e} K$ whose image $\{F\}$ in Prim $(K)$ is not open. 
We briefly indicate how such a set can be constructed. Let $K_{1}$ be a compact convex set containing only one non-trivial closed split face $\left\{\sigma_{1}\right\}$, e.g., the state space of the algebra of compact operators on an infinite dimensional Hilbert space, with the unit adjoined. Let $K_{2}$ be a square. In the direct convex sum of $K_{1}$ and $K_{2}$, identify $\sigma_{1}$ with a corner $\sigma_{2}$ of $K_{2}$. More precisely, $K$ is the state space of the order unit space

$$
A=\left\{\left(a_{1}, a_{2}\right) \in A\left(K_{1}\right) \oplus A\left(K_{2}\right):\left\langle a_{1}, \sigma_{1}\right\rangle=\left\langle a_{2}, \sigma_{2}\right\rangle\right\} .
$$

Then the only non-trivial closed split face of $K$ is (the image of) $K_{2}$, and any corner of $K_{2}$ other than $\sigma_{2}$ is isolated in $\partial_{e} K$.

\section{The primitive ideal space of a JB-algebra.}

In this section $A$ will be a JB-algebra with a unit 1 , and $K$ its state space.

We define the primitive ideal space of $A$ to be $\operatorname{Prim}(A)=\left\{\operatorname{ker} \varphi_{\varrho}: \varrho \in \partial_{e} K\right\}$, where $\varphi_{\varrho}: A \rightarrow A_{\varrho}$ is the dense representation associated with $\varrho$. (See [3; § 2]). Note that $\varphi_{Q}^{*}$ maps the normal state space of $A_{\varrho}$ bijectively onto the smallest split face $F_{\varrho}$ of $K$ containing $\varrho$. By Theorem $2.4, \bar{F}_{\varrho}$ is a split face, and indeed by the proof of that theorem, $\bar{F}_{Q}=\left(\operatorname{ker} \varphi_{e}\right)^{\perp}$. Thus $\operatorname{ker} \varphi_{Q} \rightarrow \bar{F}_{Q}$ is a bijection of $\operatorname{Prim}(A)$ and Prim $(K)$. Defining the Jacobson topology on $\operatorname{Prim}(A)$ in analogy with the $C^{*}$-algebra case, we see (using Theorem 2.3) that Prim $(A)$ and Prim $(K)$ are homeomorphic.

Theorem 4.1. Let $A$ be a JB-algebra with state space $K$. The mapping $\varrho \rightarrow \operatorname{ker} \varphi_{\varrho}$ is a continuous and open map from $\partial_{e} K$ with weak*-topology onto $\operatorname{Prim}(A)$.

Proof. We shall prove that $K$ satisfies the requirements of Theorem 3.1.

We start with Størmer's axiom. If $F_{\alpha}$ is a closed split face of $K$, the KreinMilman theorem implies that $F_{\alpha}$ is the closed convex hull of the union of all $F_{Q}$, where $\varrho \in \partial_{e} F_{\alpha}$. Thus, we need only assume given a subset $C$ of $\hat{K}=\left\{F_{e}: \varrho \in \partial_{e} K\right\}$, and we have to prove that $\overline{c o} \bigcup_{F \in C} F$ is a split face.

In [6; Cor. 5.8] it is proved that the $\sigma$-convex hull of $\partial_{e} K$, defined as

$$
\sigma-\operatorname{co}\left(\partial_{e} K\right)=\left\{\sum_{j=1}^{\infty} \lambda_{j} \varrho_{j}: \lambda_{j} \geqq 0, \sum \lambda_{j}=1, \varrho_{j} \in \partial_{e} K\right\}
$$

is a split face of $K$. We claim that $\sigma-\operatorname{co}\left(\partial_{e} K\right)$ is a direct $\sigma$-convex sum of the split faces $G \in \hat{K}$. By this we mean that any $\varrho \in \sigma-\operatorname{co}\left(\partial_{e} K\right)$ is uniquely representable in the form

$$
\varrho=\sum_{F \in \hat{K}} \lambda_{F} \varrho_{F},
$$

where $\lambda_{F} \geqq 0, \sum \lambda_{F}=1$, and $\varrho_{F} \in F$. We omit the trivial proof. (At one stage one has to use that $F^{\prime}$ is norm closed, so that $\left.\left(1-\lambda_{F}\right)^{-1} \sum_{G \neq F} \lambda_{G} \varrho_{G} \in F^{\prime}\right)$. 
Returning to our subset $C$ of $\hat{K}$, we find at once from the decomposition (4.1) that $\sigma-\operatorname{co}(\bigcup\{F: F \in C\})$ is a split face of $\sigma-\operatorname{co}\left(\partial_{e} K\right)$, and hence of $K$. By Theorem 2.4, its closure $\overline{c o}(\bigcup\{F: F \in C\})$ is also a split face, so the validity of Størmer's axiom is proved.

Next, if $\varrho \in \partial_{e} K$ then $F_{e}=\sigma-\operatorname{co}\left(\partial_{e} F_{e}\right)$, and so $\bar{F}_{e}=\overline{\operatorname{co}}\left(\partial_{e} F_{\varrho}\right)$. By Milman's theorem, $\partial_{e} F_{e}$ is dense in $\partial_{e} \bar{F}_{e}$. However, if $\sigma \in \partial_{e} F_{e}$ then $F_{\sigma}=F_{e}$, so $\bar{F}_{\sigma}=\bar{F}_{Q}$. From this the condition $\left({ }^{*}\right)$ of Theorem 3.1 follows, and the proof is complete.

Corollary 4.2. If $A$ is a JB-algebra then Prim $(A)$ is a Baire space in the Jacobson topology.

Proof. By [1; Cor. I.5.14] $\partial_{e} K$ is a Baire space in the weak*-topology. The Corollary now follows from Theorem 4.1 .

\section{REFERENCES}

1. E. M. Alfsen, Compact convex sets and boundary integrals, Ergebnisse der Math. 57, SpringerVerlag, Berlin - Heidelberg - New York, 1971.

2. E. M. Alfsen and T. B. Andersen, Split faces of compact convex sets, Proc. London Math. Soc. 21 (1970), 415-441.

3. E. M. Alfsen, H. Hanche-Olsen and F. W. Shultz, State spaces of $\mathrm{C}^{*}$-algebras, Acta Math. (to appear).

4. E. M. Alfsen and F. W. Shultz, Non-commutative spectral theory for affine function spaces on convex sets, Mem. Amer. Math. Soc. 172 (1976).

5. E. M. Alfsen and F. W. Shultz, On non-commutative spectral theory and Jordan algebras, Proc. London Math. Soc. 38 (1979), 497-516.

6. E. M. Alfsen and F. W. Shultz, State spaces of Jordan algebras, Acta Math. 140 (1978), 155190.

7. E. M. Alfsen, F. W. Shultz and E. Størmer, A Gelfand-Neumark theorem for Jordan algebras, Advances in Math. 28 (1978), 11-56.

8. C. M. Edwards, Ideal theory in JB-algebras, J. London Math. Soc. 16 (1977), 507-513.

9. C. M. Edwards, On the facial structure of a JB-algebra, J. London Math. Soc. 19 (1979), 335344.

10. E. G. Effros, Order ideals in a C*-algebra and its dual, Duke Math. J. 30 (1963), 391-412.

11. E. G. Effros and E. Størmer, Jordan algebras of self-adjoint operators, Trans. Amer. Math. Soc. 127 (1967), 313-316.

12. J. Glimm, Type I C*-algebras, Ann. of Math. 13 (1961), 572-612.

13. H. Hanche-Olsen, $A$ note on the bidual of a JB-algebra, Math. Z. (to appear).

14. R. Prosser, On the ideal structure of operator algebras, Mem. Amer. Math. Soc. 45 (1963), 1-28.

15. F. W. Shultz, On normed Jordan algebras which are Banach dual spaces, J. Functional Analysis 31 (1979), 360-376.

16. E. Størmer, On partially ordered vector spaces and their duals, with applications to simplexes and C*-algebras, Proc. London Math. Soc. 18 (1968), 245-265. 